\title{
On the Bone Tumours: Overview, Classification, Incidence, Histopathological Issues, Behavior and Review Using Literature Data
}

\author{
Alina Maria Sisu, Loredana Gabriela Stana, Codruta lleana Petrescu, \\ Romulus Fabian Tatu, Roxana Folescu and Andrei Motoc
}

Additional information is available at the end of the chapter

http://dx.doi.org/10.5772/52969

\section{Introduction}

\subsection{Classification of bone tumours}

According to World Health Organization, bone tumours can be divided into primary and secondary, [7]. Primitive bony tumours are classified using histo-genetic criteria and malignancy anatomic-clinical criteria.

\subsubsection{Tumours that form bones}

Benign: osteoma, osteoid osteoma, benign osteoblastoma;

Malignant: osteosarcoma (osteogenic sarcoma) with subtypes: conventional, chondroblastic, fibroblastic, osteoblastic, telangiectatic, small cell, low-grade central, secondary, parosteal, periosteal, high-grade surface,[8].

\subsubsection{Tumours that form cartilage}

Benign: chondroma, osteochondroma, chondroblastoma, chondromixoid fibroma;

Malignant: chondrosarcoma with subtypes: central, primary and secondary, peripheral, dedifferentiated, mesenchymal, clear cell;

a. Medullar tumours: Ewing sarcoma/ primitive neuroectodermal tumour;

It is the third most common bone cancer. Most Ewing tumors start in bones, but they can start in other tissues and organs. This cancer is most common in children and teenagers. It is rare in adults over age 30. 
b. Giant cell tumours, malignant giant cell tumours, osteoclastoma;

c. Fibrogenic tumours: fibrosarcoma;

d. Fibrohistiocystic tumours: malignant fibrous histiocytoma;

e. Haematopoietic tumours: plasma cell myeloma, malignant lymphoma;

f. Notochordal tumours: chordoma;

g. Vascular tumours: angiosarcoma;

h. Smooth muscle tumours: leiomyosarcoma;

i. Lipogenic tumours: liposarcoma;

j. Miscellaneous tumours: adamantinoma;

\begin{tabular}{|l|l|l|l|}
\hline Tumour type & Age & Location & Histologic aspect \\
\hline Osteoma & $41-50$ & Skull bones & Matured lamellar bone \\
\hline Osteoid osteoma & $11-20$ & $\begin{array}{l}\text { Short and long } \\
\text { bones diaphysis }\end{array}$ & $\begin{array}{l}\text { Osteiod outlined by osteoblasts, } \\
\text { incorporated in a fibrous stroma }\end{array}$ \\
\hline Osteosarcoma & $10-25$ & $\begin{array}{l}\text { Long bones } \\
\text { metaphysis }\end{array}$ & $\begin{array}{l}\text { Osteoid and bone formed of } \\
\text { malignant osteoblasts and } \\
\text { fibroblasts. }\end{array}$ \\
\hline Chondroma & $11-40$ & Feet, hands & $\begin{array}{l}\text { Maturated hyaline cartilage } \\
\text { (enchondroma/ecchondroma), } \\
\text { preserving lobulation }\end{array}$ \\
\hline Chondrosarcoma & $30-60$ & $\begin{array}{l}\text { Long bones } \\
\text { metaphysic, } \\
\text { axial skeleton }\end{array}$ & $\begin{array}{l}\text { Immature cartilage, no } \\
\text { preserving lobulation, cells } \\
\text { arranged in groups of two or } \\
\text { four, with atypia and mitosis }\end{array}$ \\
\hline Ewing sarcoma & $5-25$ & $\begin{array}{l}\text { Long bones } \\
\text { diaphysis }\end{array}$ & $\begin{array}{l}\text { Small, round, undifferentiated } \\
\text { cells, no stroma, a lot of capillary } \\
\text { arrangement. }\end{array}$ \\
\hline Giant cells tumour & $20-40$ & Knee & $\begin{array}{l}\text { Multinucleated giant cells, } \\
\text { fusiform cells, mononuclear } \\
\text { cells. }\end{array}$ \\
\hline Metastases & $50-90$ & Anywhere & Frequently adenocarcinomas \\
\hline
\end{tabular}

Table 1. Overview on tumours

Our study revealed 198 cases of benign tumours, with a male/female ratio=1.2/1, with an average age of 41 years, ages between 15-78. The most afected were 21-30 and 51-60 age groups. A male predominance in males in 21-30 group was revealed. In 11-40 age group were highlighted 69 out of 108 cases $(63.88 \%)$. In 51-60 age group was a female predominance, 27 cases. The most frequent osseous benign tumour in our study was osseous cyst followed by giant cell tumour(Table 1).

In 2009-2011 in our clinic we treated 87 tumour osseous cases. Out of these, 19 were treated using surgical biopsy and 68 were entirely excised. Sites, morphological types of the bone tumours stated during the histopathological examination and their frequency are shown in Tables 2, 3 . 


\begin{tabular}{|l|l|}
\hline Anatomical site & Number of cases \\
\hline Hip bone & 6 \\
\hline Proximal extremity of femur & 8 \\
\hline Distal extremity of femur & 13 \\
\hline Fibula & 3 \\
\hline Proximal extremity of tibia & 8 \\
\hline Middle 1/3 of tibia & 7 \\
\hline Distal 1/3 of tibia & 3 \\
\hline Synovial cyst of the leg (synovialoma) & 9 \\
\hline Superior surface of the foot & 4 \\
\hline Radiocarpal cyst & 18 \\
\hline Humerus & 5 \\
\hline Proximal 1/3 ulna & 3 \\
\hline
\end{tabular}

Table 2. Anatomical sites of the bone tumours

\begin{tabular}{|l|l|}
\hline Tumour & Number of cases \\
\hline Epidermal cyst & 4 \\
\hline Synovialoma & 9 \\
\hline Osteochondroma & 49 \\
\hline Osteoclastoma & 7 \\
\hline Osteosarcoma & 7 \\
\hline Lipomiosarcoma & 1 \\
\hline Giant cell tumour & 8 \\
\hline Solitary osseous mieloma & 2 \\
\hline
\end{tabular}

Table 3. Histopathological examination

\subsection{Benign tumours}

\subsubsection{Solitary osseous cyst}

From microscopically point of view, is a dense osseous tissue which outlines a well -blood supplied connective tissue, sometimes macrophages filled with hemosiderin and colesterol.

\subsubsection{Giant cell tumour or mieloplaxe tumour or osteoclastoma}

Is composed of mononucleated stroma, with fusiform cells, well- blood supplied, and of giant multinucleated cells, resembling osteoclasts. Microscopically cells are multinucleated, giant, having a mesenchymal origin, with dimensions 10-50 microns, with 20-30 nuclei central situated, in a basophile cytoplasm and a fibrous stroma.

\subsubsection{Osteoid osteoma}

Is a solitary benign tumour which produces dense osseous tissue with a particular entity, nidus. Microscopically, the central zone contains osteoid tissue with osteoblasts, osteoclasts 
and fibroblasts, in a network of well -blood supplied osteoid travee. These are anastomosed each other and have a progressive calcification, making a final image of osteosclerosis.

\subsubsection{Osteoma}

Is a unique or multiple benign tumour, formed by bones osteoforming proliferation with membranous origin. Microscopically is slighty different from adult osseous tissue. It has irregular osseous travee, located around the haversian spaces.

On 198 cases of benign tumours discovered and treated in Romania, we had the following distribution: solitary osseous cyst 66 cases, giant cell tumour 63 cases, osteoid osteoma 36 cases, osteoma 33 cases.

\subsection{Malignant tumours}

\subsubsection{Osseous metastases incidence}

In Sweden, in 10 years from a group of 832 cases of malignant primary bone tumours 242 were osteogenic sarcoma (28.8\%), 193 chondrosarcoma $(22.9 \%)$ and 74 cases Ewing's sarcoma (8.8\%). All three tumours showed a predilection for males,[9].

In Ethiopia in 2003-2008 were treated 216 bone tumour patients with a male/female ratio=1. Of these, $36 \%$ (74/205) were malignant. The commonest was osteosarcoma, 52/182, 28.5\%, [10].

According to Marugame et al,[11], the distribution of histological type for primary bone cancer in Japanese populationfor 1993-2001 was: osteosarcoma, the most frequenthistological type, accounting for approximately $40 \%$. Chondrosarcoma was the second-most frequent, accounting for approximately 25\%. Ewing sarcoma was the thirdmost frequent, accounting for approximately $10 \%$. Malignant fibrous histocytoma and giant cell tumor accounted for approximately 6 and $2 \%$, respectively.

In North America and Europe, the incidence rate for bone sarcomas in males is approximately 0.8 new cases/100,000 populations. Higher incidence rates have been observed on males in Argentina and Brazil (1.5-2/1=M/F) and Israel (1.4/1=M/F). From histological point of view osteosarcoma is the most common primary malignant tumour of bone, accounting for approximately $35 \%$, chondrosarcoma (25\%), Ewing sarcoma (16\%),[12].

The most frequent cancers that give osseous metastases are: breast carcinoma, small cell pulmonary carcinoma, renal carcinoma, thyroid carcinoma, prostate carcinoma.

Once the tumour metastases in the bone it becomes incurable. $20 \%$ of patients suffering from breast cancer live 5 years after discovering a bony metastasis. Breast and prostate cancers spread especially in bones.

Osteosarcoma is the most frequent malignant primary bone tumour, with a higher incidence in 15-20 year old group. Male/female ratio is 1.4-1.5-1. Ewing sarcoma is the second most common primary malignant bone cancer, seen most frequently on children and adolescents. 
Chondrosarcoma occurs mostly in adulthood, with a male/female ratio=1. Our data showed an increased percentage in males in Romania, but a 3/1 female/male ratio in Timisoara,[13].

\subsubsection{Osteosarcoma}

Site and incidence

Osteosarcoma is the most common primary malignant tumour of bone, more common in males. The incidence is $3 / 1,000,000$ population. It accounts for $<1 \%$ of all malignant neoplasm. The most frequent site is the distal femur, followed by the proximal tibia and the proximal humerus.

In Romania field 2005-2010 were treated 468 cases of osteosarcomas with a male/female ratio $=1.3 / 1$, with some variability in clinics (in our clinic 7 out of 18 cases were osteosarcomas. Ratio $\mathrm{F} / \mathrm{M}=3 / 1$.) Out of these 468, 198 were benign (42.30\%) and 189 malignant (40.38\%). Out of these malignant, with a male/female ratio $=1.2 / 1,168$ were malignant $(88.88 \%)$. As benign tumours on the first place was osseous cyst and secondary the giant cell tumour. As age groups, 21-30 and 51-60 years were equal, 45 case each, with a significant difference: in the first group 27 cases were females and in the last one 27 cases were males.

From primary malignant tumours point of view a ratio male/female=1.33/1. Most of these tumours after the histopathological examination were osteosarcomas.

From secondary malignant tumours point of view a ratio male/female=1/1. 51 were carcinomas, 42 malignant fibrous histiocytomas, and 27 fibrosarcomas.

Locations of osteosarcomas are: osseous, central, surface, gnathic, multifocal, soft tissue, intramuscular.

The most frequent location was femur (50\%), followed by tibia $19.6 \%$, humerus $15.2 \%$ and distal fibula $2.2 \%$.

Accidentally, osteosarcomas could be found in hyoid bone or nasal septum.

Histology - Microscopically types

- Central: high-grade, conventional, telangiectatic, small cell, epitheloid, osteoblastomalike, chondroblastoma-like, fibrohistiocystic, giant cell;

- Low-grade: low-grade central, fibrous dysplasia-like, desmoplastic fibroma-like;

Surface: low-grade, parosteal, intermediate-grade, periosteal, high-grade, dedifferentiated parosteal, high-grade surface;

- Intracortical;

- Gnathic;

- Extraskeletal: high-grade, low-grade;

Diferent types

- Conventional Osteosarcoma is also divided into osteoblastic, chondroblastic and fibroblastic subtypes according to histological feature, even from treatment and 
response point of view there is no difference between them. Grading the osteosarcoma is important from oncologic point of view, because based on this could be found the best treatment, especially the type of surgery.

Using Broders schema, the grade of tumour is numbered from 1 to 4 , depending on the percentage of anaplasia, the cytologic atypia of the cells being the most important factor in grading tumours (Figure 1).

- Telangiectatic Osteosarcoma is an osteosarcoma in which take place local destructions with replacement of anatomic spaces. New formed aneurismal bone cyst and production of osteoid bone can establish diagnosis.

- Giant cell-richosteosarcomas contain osteoclast-like giant cells.

- Small cell osteosarcoma represents a rare histological combination of osteosarcoma and Ewing sarcoma, until $2 \%$ of osteosarcomas.

- Epithelioid osteosarcoma has the cell tumour poorly differentiated, for this reason being difficult to distinguish if is a sarcoma or a carcinoma.

- Osteoblastoma-like and chondroblastoma-like osteosarcoma resembles osteoblastoma with atypical osteoblasts and having different histological feature. These tumours are extremely rare, but are important to be established a precise diagnosis; these could metastasize (Figure 2).

- Giant cell-rich osteosarcoma contains benign multinucleated giant cells, but sometimes could contain lot of benign giant cells that cover the real malignant elements (Figure 3).

- Gnathic osteosarcomas appear in maxilla and mandible bone. They are chondroblastic, osteoblastic, fibroblastic, small cell type concerning the matrix production.

- Low-grade central osteosarcomas have been reported as very rarely, resembling the low-grade parosteal sarcoma, fibrous dysplasia and other benign lesions (Figure 4).

- Surface osteosarcomas consist of osteosarcoma whose epicentres are out of the cortex of the bone outlines. According to some criteria (anatomic location, predominant pattern of matrix, histological grade) there are several types of surface osteosarcomas: parosteal osteosarcoma, periosteal osteosarcoma, dedifferentiated parosteal osteosarcoma, high-grade surface osteosarcoma.

i. Parostealosteosarcoma is the most common form of surface osteosarcomas, frequently been confused with osteochondroma and osteoma. Is credited with $<0.5 \%$ of osteosarcomas, $70-83 \%$ out of them are located on distal posterior femur.

ii. Periosteal osteosarcoma is rarely than parosteal osteosarcoma and has a cartilaginous matrix component. As histological grade is between I grade parosteal osteosarcoma and III/IV grade osteosarcoma.

iii. Dedifferentiated parosteal osteosarcoma is composed of low-grade parosteal osteosarcoma and high-grade conventional parosteal osteosarcoma. According to Rizzoli Institute, dedifferentiation occurs in $25 \%$ of low-grade parosteal osteosarcomas.

iv. High-grade surface osteosarcoma is microscopically high-grade. It could be possible to have a high-grade surface osteosarcoma that is a dedifferentiated parosteal osteosarcoma in which the high-grade component has replaced the low-grade component.

- Intracortical osteosarcoma is very rare high-grade osteosarcoma that from histological point of view is osteoid or maybe bone formation. It is treated like conventional osteosarcoma (Figures 5,6). 
- Multifocal osteosarcoma is unusual, affect children, young adults. It is a high-grade sarcoma, very aggressive, without escape in terms of surviving.

- Extraskeletal osteosarcoma is credited with $<2.2 \%$ of all soft tissue sarcomas. From histological point of view it resembles all types of osteosarcoma, even it has grown as soft tissue in low-grade central osteosarcoma. $3 / 4$ of patients are dying in the first 5 years of diagnosis.

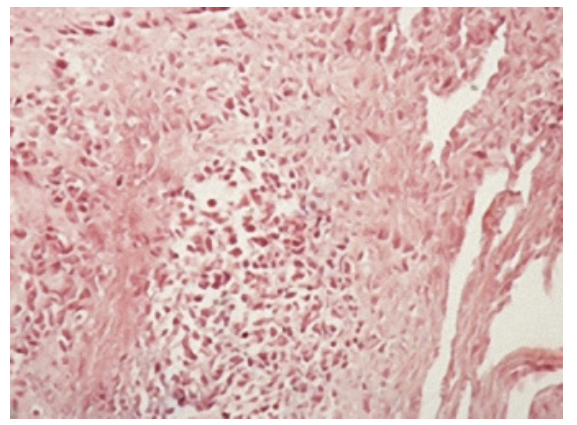

Figure 1. Conventional osteosarcoma with abundance of hyper chromatic nuclei, polyhedral tumour cells, sarcomatous vessels; HE staining X 100 (microscopic aspect)

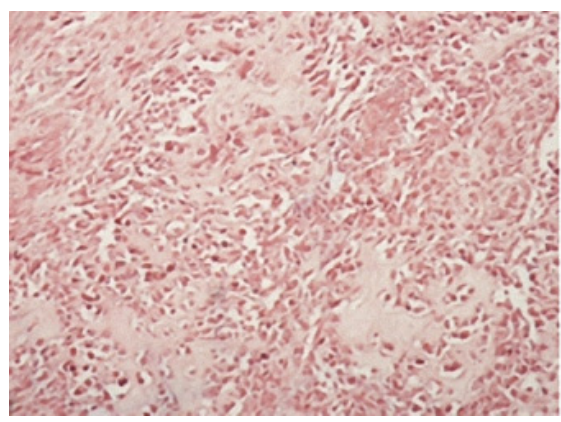

Figure 2. Chondroblastic osteosarcoma - compact groups of malignant tumour cells, areas with cellular hyaline cartilage and osteiod formation; HE staining X 100 (microscopic aspect)

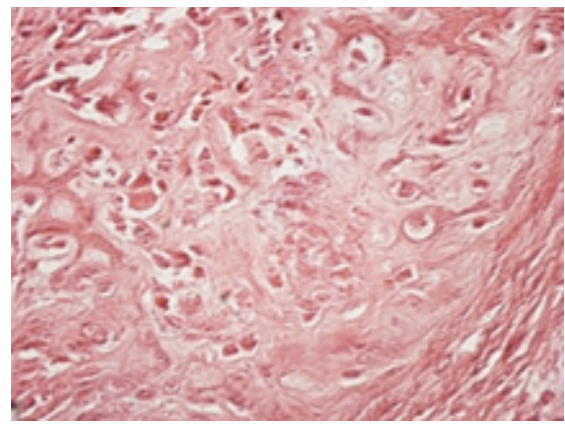

Figure 3. Classic osteosarcoma with an abundant production of tumour osteoid areas and bone matrix, enclosing giant malignant tumour cells; HE staining X400 (microscopic aspect) 


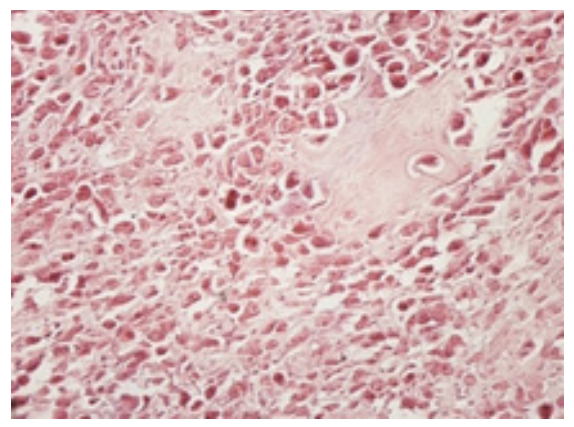

Figure 4. Osteoid osteosarcoma- polyhedral tumour cells, with atypical mitosis, little bone and osteoid matrix; HE staining X 200 (microscopic aspect)

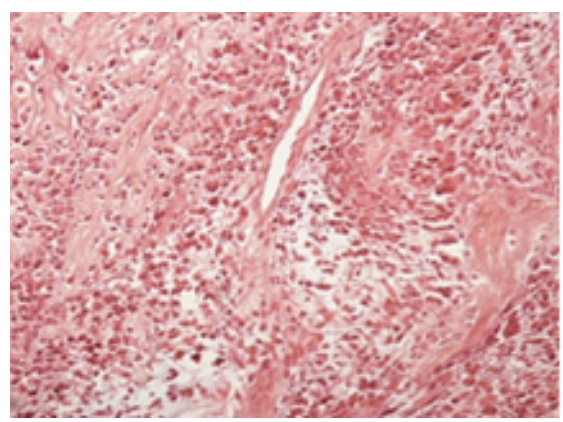

Figure 5. Osteosarcoma-tumour cells having sizes and shapes variable with hyper chromatic nuclei and mitosis areas; HE staining X 100 (microscopic aspect)

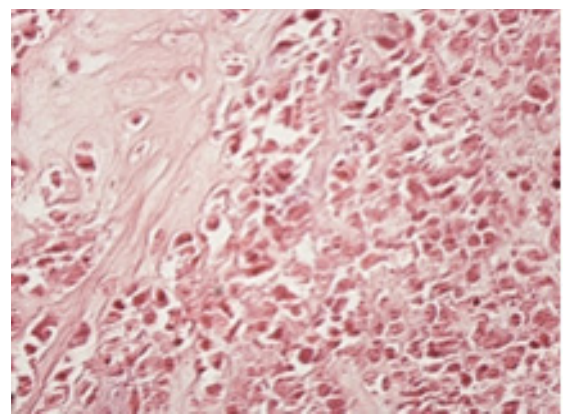

Figure 6. Osteosarcoma- polymorph tumour cells having a big size nucleus, prominent nucleolus and osseous matrix; HE staining X 200 (microscopic aspect)

\subsubsection{Staging bone tumours}

As Enneking et al have stated,[14], there is a system for staging bone sarcomas, according to correlation of the tumour location and metastases presence(Table 4);T1 - the tumour is intra compartmental; T2 - the tumour is extra compartmental; M0 - no regional or distant metastasis; M1 - regional or distant metastasis; G1 - low grade; G2 - high grade. 


\begin{tabular}{|l|l|l|l|}
\hline Stage & Tumour & Metastases & Grade \\
\hline I A & T1 & M0 & G1 \\
\hline I B & T2 & M0 & G1 \\
\hline II A & T1 & M0 & G2 \\
\hline II B & T2 & M0 & G2 \\
\hline III & T1 or T2 & M1 & G1 or G2 \\
\hline
\end{tabular}

Table 4. Enneking staging system for primary malignant tumours of bone

According to American Joint Committee on Cancer Staging System $[15,16]$ it has been used a new, more complex classification of the primary malignant osseous tumours, where also is taken into consideration the lymphatic nodes existence (Table 5), a criterion which states the following:Tx - primary tumour cannot be assessed; T0 - no evidence of primary tumour; T1 tumour $8 \mathrm{~cm}$ or less in greatest dimension; T2 - tumour more than $8 \mathrm{~cm}$ in greatest dimension; T3 - discontinuous tumours in the primary bone; $\mathrm{Nx}$ - regional lymph nodes not assessed; N0 - no regional lymph node metastases; N1 - regional lymph node metastases; Mx - distant metastasis cannot be assessed; M0 - no distant metastasis; M1 - distant metastasis; M1a - lung; M1b - other distant site; Gx - grade cannot be assessed; G1 - well differentiated (low grade); G2 - moderately differentiated (low grade); G3 - poorly differentiated (high grade); G4 - undifferentiated (high grade).

\begin{tabular}{|l|l|l|l|l|}
\hline Stage & Tumour & Lymph Node & Metastases & Grade \\
\hline IA & T1 & N0 & M0 & G1 OR G2 \\
\hline IB & T2 & N0 & M0 & G1 OR G2 \\
\hline IIA & T1 & N0 & M0 & G3 OR G4 \\
\hline IIB & T2 & N0 & M0 & G3 OR G4 \\
\hline III & T3 & N0 & M0 & ANY G \\
\hline IVA & ANY T & N0 & M1a & ANY G \\
\hline IVB & ANY T & N1 & ANY M & ANY G \\
\hline IVB & ANY T & ANY N & M1b & ANY G \\
\hline
\end{tabular}

Table 5. Staging of the primary malignant osseous tumours

\subsubsection{Chondrosarcoma}

Site and incidence

In 1994-2007 were assessed 62 cases of chondrosarcomas in Romania, with a slightly decreasing for the next two years.

Out of these 62 patients 46 were males (74.2\%) and 16 females (25.8\%). Male/female ratio $=2.88 / 1$. On age groups distribution was the following: average age of all patients was 48.8 years, 16-81. On gender groups' distribution was the following: average age in females was 59.10, 16-78; average age in males was 45.26, 16-71. On gender and age groups the highest frequency is on $45-56$ years in males, almost $20 \%$. $66-75$ group age in females, $9.8 \%$. 
As example, in 2001-2007, in Russian Federation were examined 77 patients with chondrosarcoma. The dedifferentiated form of the tumor was confirmed in 10 (13\%) cases.

The most common place is femur, $41.9 \%$, followed by tibia, $16.1 \%$ and humerus $9.7 \%$. Less frequent chondrosarcoma is highlighted in hip bone, $16.2 \%$, phalanges $6.5 \%$, and $3.1 \%$ in calcaneus, scapula and vertebrae. From $41.9 \%$ chondrosarcomas located on the femur $53.8 \%$ has a distal location. On tibia and humerus the location of a chondrosarcoma is $100 \%$ proximal.

Histology

From histological feature point of view, chondrosarcomas are divided in following groups:

- Well differentiated chondrosarcoma (differential diagnosis with rich-cell chondroma);

- Clear cell chondrosarcoma (with a "broken glass" cytoplasm);

- Myxoid chondrosarcoma - II grade (differential diagnosis with chondromyxoid fibroma);

- Dediferentiated chondrosarcoma (has a different sarcomatous area);

- Mesenchymal chondrosarcoma.

In order to have a precise diagnosis are followed some criteria: cellular density, cellular atypia, mitosis, according to these being described the grade of malignancy. Radiologic imaging is the first that could put a screening diagnostic, followed by MRI and RMN.

Magnetic Resonance Imaging (MRI) can be helpful in differentiating between benign and malignant lesions in several ways. Greater than $90 \%$ medullar involvement can be suggestive of chondrosarcoma, while the absence of $90 \%$ medullar involvement of noncontiguous areas of cartilage within the bone can suggest the presence of an enchondroma.

In addition, the timing and progression of gadolinium contrast enhancement patterns may help direct a clinician toward or away from a diagnosis of malignancy. Many surgeons consider MRI critical for surgical planning because it can illustrate the tumour extension involved in bone and soft tissues $[17,18]$.

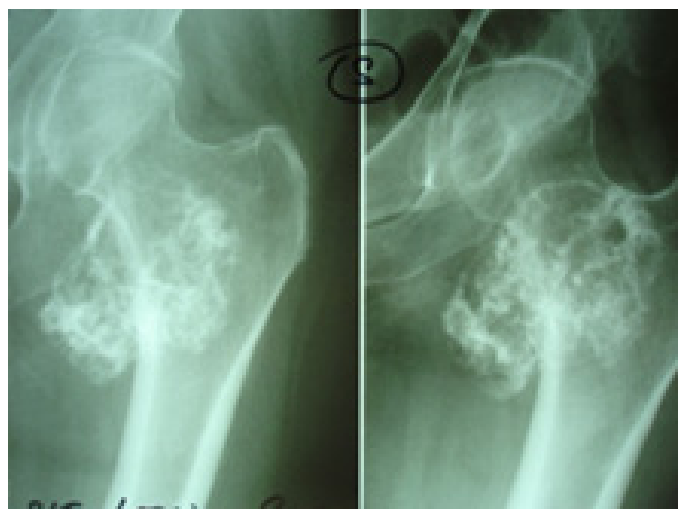

Figure 7. Large hip joint tumour on the inferior surface of the lesser trochanter of the femur (radiologic images) 


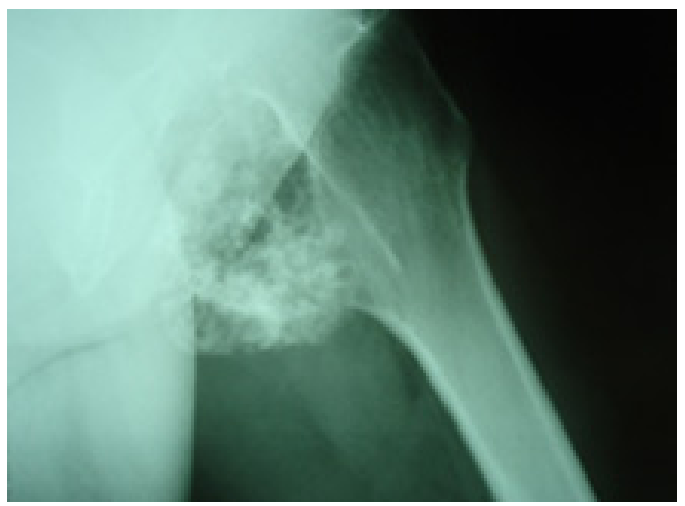

Figure 8. X- ray: Hip joint tumour, with clear, obvious, inhomogeneous outline

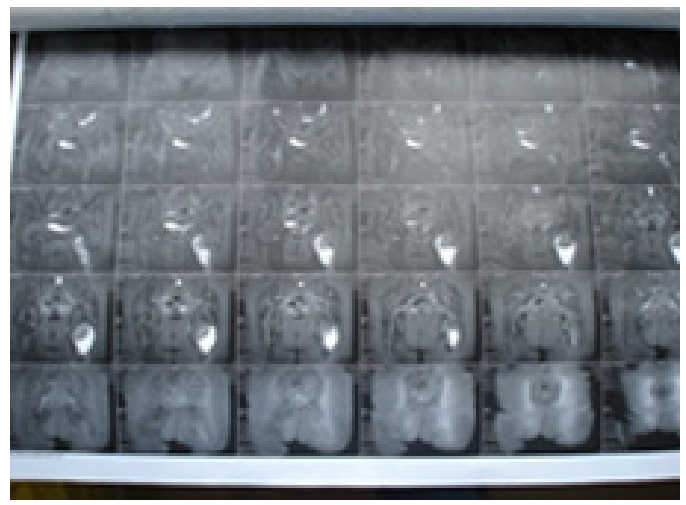

Figure 9. MRI sagittal section: shows an important tumour, 80/50/60 mm with a lot of liquid

On macroscopic examination, chondrosarcoma is seen like a grayish-white, lobulated mass. It has focal calcification and muriform aspect (Figure 10). The bigger one $(2 / 1,5 \mathrm{~cm})$ is decalcified.

Histological, the tumour is stained HE. Could be found tumour fragments with lobulated pattern composed of cartilage matrix which supports many chondroplasts congested with focal loss of arranging symmetrical character and containing not a strong polymorphism (Figures 11, 12, 13, 14).

It was associated blades oblong of bone compact tissue. These were deformed and fragmented by the invasion of tumour tissue. Histological aspects are in favour for welldifferentiated chondrosarcoma, [19-21].

\subsection{Survival rate}

Based on the literature data for 1995-2001, the overall 5-year relative bone cancer survival rate was calculated $69.4 \%$. By rase and gender groups it was: $67.5 \%$ for Caucasian men; 
72.1\% for Caucasian women; $70 \%$ for Afro-American men; $68.4 \%$ for Afro-American women.

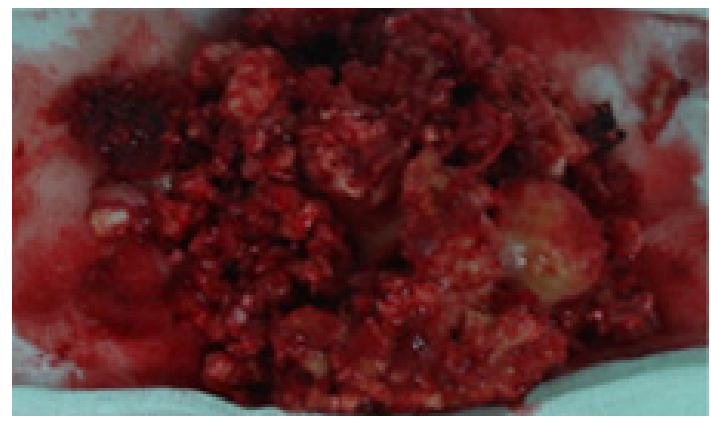

Figure 10. Macroscopic aspect; exophytic sarcoma, with calcified areas and haemorrhage

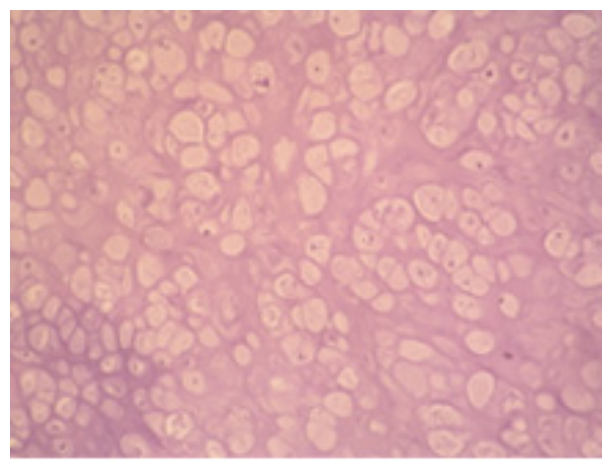

Figure 11. Well-differentiated chondrosarcoma consisting of pale hyaline matrix; HE staining X 40 (microscopic aspect)

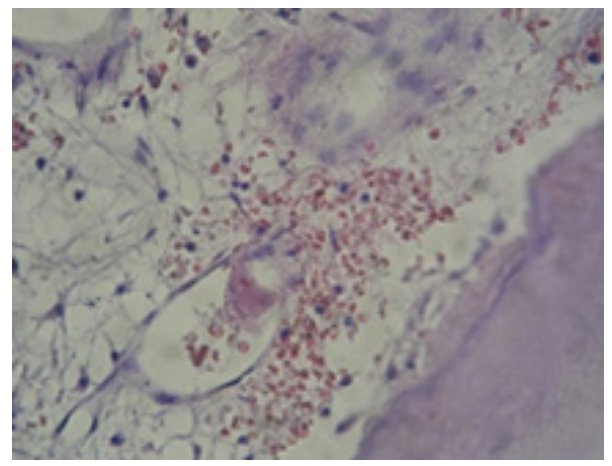

Figure 12. Malignant chondrocytes, large, atypical, with large nuclei; HE staining X 40(microscopic aspect) 


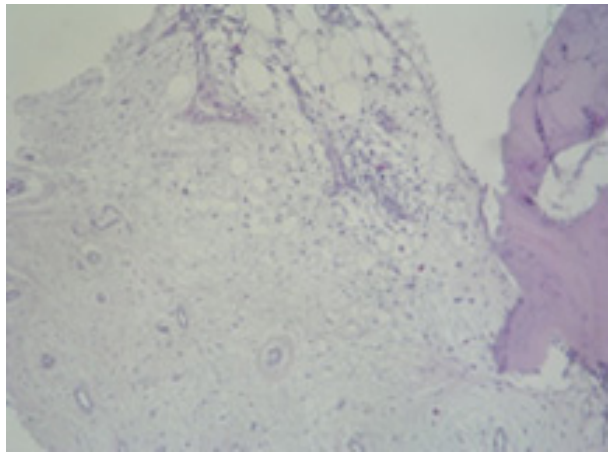

Figure 13. Well-differentiated chondrosarcoma consisting of nodules hyaline matrix; lymphoplasmocytes infiltrate; HE staining x 10 (microscopic aspect)

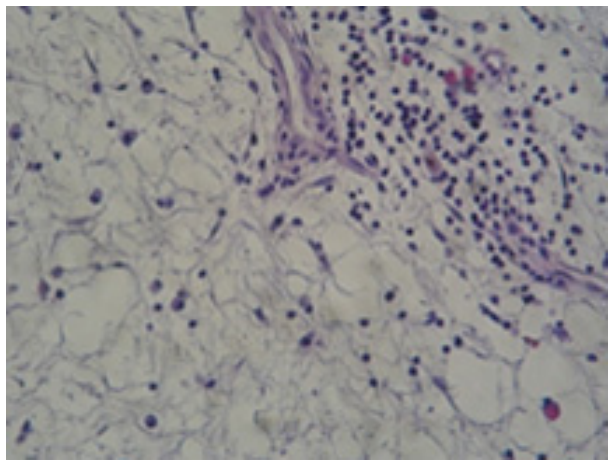

Figure 14. Well-differentiated chondrosarcoma consisting of nodules hyaline matrix; lymphoplasmocytes infiltrate; HE staining X 40 (microscopic aspect)

\subsection{Bone cancer statistics on stages}

This is very important for the prognosis.

- $41 \%$ of bone cancer cases are diagnosed while the cancer is still confined to the primary site, so it is a localized stage.

- $\quad 36 \%$ of bone cancers are diagnosed after the cancer has spread to regional lymph nodes or directly beyond the primary site.

- $15 \%$ of bone cancer cases are diagnosed after the cancer has already metastasizes, so it is a distant stage.

- $8 \%$ of bone cancer cases had staging unknown.

- In literature cases the corresponding 5-year relative bone cancer survival rates were:

- $84.5 \%$ for localized stage; $69.4 \%$ for regional stage; $30.6 \%$ for distant stage; $62.2 \%$ for unknown stage. 


\section{Conclusions}

The most important thing in dealing with a bone tumour is a correct and full diagnosis. This include: clinical staging, a right excision, with $5 \mathrm{~cm}$ limits around tumour, a very precise histopathological examination and, not for the last, a post surgery treatment (radiotherapy, hormonal therapy, immunotherapy, chemotherapy). All these have in common an increase of 5-year survival rate.

Taking into account that malignant primary bone tumours are few, the secondary ones, meaning the metastases, are the dangerous. So, besides the treating of the metastases, is also essential to treat, and sometimes to find, the primary tumour. It is very true that the secondary tumour is discovered when the primary is in an advanced stage and the rate survival decreases very much.

Metastases behaviour is different from the primary tumour behaviour. Histopathological feature is different on breast tumours successive metastases, suggesting molecular changes depending on the tissue where the tumour is growing. Tumour cells preserve the initial pattern of the origin tissue, but the malignant phenotype is modified, depending on the metastazing area,[22].

Conventional radiography is very useful for diagnostic information. Magnetic resonance imaging (MRI) is recommended over computed tomography (CT) scanning for delineation of tumour extent before surgery.

Nuclear imaging is limited in providing diagnosis for bony lesions. Angiography is useful when a compression on the vessels is suspected. Of course, is also important the location of the tumour on the bone.

Histopathological examination of the biopsy sample provides with certainty the type of the tumour, but in some cases the tumour feature so resembles to others that is very difficult, even for an old specialist, to put without any doubt, a correct diagnosis.

A longer survival of cancer patients leads to a higher risk of population to develop bone metastases and pathological fractures. For this reason, reconstructive procedure requires a guarantee longer term, in order to avoid mechanical problems during the life of the patient,[23]. The follow-up of the patients is multidisciplinary, including oncology, orthopaedics, radiology, geriatrics, endocrinology, intensive care, physiokinetotherapy.

\section{Author details}

Alina Maria Sisu, Loredana Gabriela Stana, Codruta Ileana Petrescu, Roxana Folescu and Andrei Motoc

Department I Anatomy and Embryology, Faculty of Medicine, "Victor Babes"University of Medicine and Pharmacy Timisoara, Romania

Romulus Fabian Tatu

Department of Orthopaedics, Traumatology, Urology and Imagistics, Faculty of Medicine, "Victor Babes"University of Medicine and Pharmacy Timisoara, Romania 


\section{References}

[1] Von Schulthess Ch, Zollikofer L. In G.K. von Schulthess Ch.L. Zollikofer, editor, Musculoskeletal Diseases Springer Milan Berlin Heidelberg, New York; 2005.

[2] Coleman RE, Rubens RD. The Clinical Course of Bone Metastases from Breast Cancer. Breast Journal Cancer 1987; 55:61-66.

[3] Anderson BO, Shyyan R, Eniu A et al. Breast Cancer in Limited-Resource Countries: An Overview of the Breast Health Global Initiative 2005 Guidelines. Breast Journal 2006; 12 S3-15.

[4] Mundy GR. Metastasis to Bone: Causes, Consequences and Therapeutic Opportunities. National Review Cancer 2002; 48(2):584-593.

[5] Cancer Stats: Worldwide Cancer London: Cancer Research UK; 2005.

[6] Cancer Stat Fact Sheets. Cancer of the Breast. Bethesda: National Cancer Institute 2005.

[7] World Health Organization. Fact Sheet No. 297 In Cancer WHO, Geneva; 2006.

[8] World Health Organization: WHOSIS (WHO Stastistical Information System) 2006: A Guide to Statistical Information at WHO- World Health Statistics 2006; http://www.who.int/whosis/en/ (accessed November, 10, 2011).

[9] Larsson SE, Lorentzon R. The Geographic Variation of the Incidence of Malignant Primary Bone Tumors in Sweden. Journal of Bone and Joint Surgery 1974; 56-A, 592600.

[10] Negash BE, Admasie D, Wamisho WE, Tinsay MW. Bone Tumors at Addis Ababa University, Ethiopia: Agreement Between Radiological and Histopathological Diagnoses, A -5-year Analysis at Black-Lion Teaching Hospital. International Journal of Medicine and Medical Science 2009; 1(4),119-125.

[11] MarugameT, Katanoda K, Matsuda T, Hirabayashi Y, Kamo K, Ajiki W and Sobue T. The Japan Cancer Surveillance Research Group, The Japan Cancer Surveillance Report: Incidence of Childhood, Bone, Penis and Testis Cancers. Japanese Journal of Clinical Oncology 2007;37(4)319-323.

[12] American Cancer Society: Information and Resources for Cancer 2000-2008.

[13] Poenaru DV, Raica M, Ouassim D. In Metastazele osoase, Editura Mirton; 2009.

[14] Enneking WF, Spanier SS, Goodman MA. A System for the Surgical Staging of Musculoskeletal Sarcoma. Clinical Orthopaedics 1980; 153:106-120.

[15] American Joint Committee on Cancer Bone. In: Fleming ID, Cooper JS, HensonDE, et al. AJCC Cancer Staging. 1997:143-147.

[16] American Joint Committee on Cancer Bone. In: GreeneFL, Page DL, Fleming ID, et al. AJCCCancer Staging Manual New York Springer- Verlag; 2002.

[17] Sandberg AA, Bridge JA. Updates on the Cytogenetics and Molecular Genetics of Bone Soft Tissue Tumors: Chondrosarcoma and Other Cartilaginous Neoplasms. Cancer Genetics Cytogenetics2003; 143:1-31.

[18] Wehrli BM, Huang W, de Cromrugghe B, Ayala AG, Czerniak B. Sox 9, A Master Regulator of Chondrogenesis, Distinguishes Mesenchymal Chondrosarcoma From Other Small Blue Round Cell Tumors. Human Pathology2003; 34:263-269. 
[19] Mitchel A, Ruda NJR, Fenton PV. Juxtacortical Dedifferentiated Chondrosarcoma from a Primary Periosteal Chondrosarcoma. Modern Pathology1996; 9:279-283.

[20] Rosenberg AE, Neilsen GP, Keel SB., Renard LG, Fitzek MM, Munzenrider JE, et al. Chondrosarcoma of the Base of the Skull: A Clinicopathologic Study of 200 Cases with Emphasis on Its Distinction from Chordoma. American Journal of Surgical Pathology1999; 23:1370-1378.

[21] Kalil RK, Inwards CY, Unni K, Bertoni F, Bacchini P, Wenger DE, et al. Dedifferentiated Clear Cell Chondrosarcoma. American Journal of Surgical Pathology 2000; 24:10791086.

[22] Peh WCG, Muttarak M. Bone Metastases. Retrieved July 27, 2005; http://emedicine.com/radio/topic88.htm (accessed December, 11, 2011).

[23] Greenberg HS, Deck MD, Vikram B, et al. Metastasis to the Base of the Skull; Clinical Finding in 43 Patients. Neurology 1981; 31:530-537. 